\title{
Exceptional responders with invasive mucinous adenocarcinomas: a phase 2 trial of bortezomib in patients with KRAS G12D-mutant lung cancers
}

\author{
Alexander Drilon, ${ }^{1,2,6}$ Adam J. Schoenfeld, ${ }^{1,6}$ Kathryn C. Arbour, ${ }^{1}$ Anna Litvak, ${ }^{1}$ \\ $\mathrm{Ai} \mathrm{Ni}{ }^{3}$ Joseph Montecalvo, ${ }^{4}$ Helena A. Yu, ${ }^{1,2}$ Elizabeth Panora, ${ }^{1}$ Linda Ahn, ${ }^{1}$ \\ Maureen Kennedy, ${ }^{1}$ Anne Haughney-Siller, ${ }^{1}$ Vincent Miller, ${ }^{1}$ Michelle Ginsberg, ${ }^{5}$ \\ Marc Ladanyi, ${ }^{4}$ Maria Arcila, ${ }^{4}$ Natasha Rekhtman, ${ }^{4}$ Mark G. Kris, ${ }^{1,2}$ \\ and Gregory J. Riely ${ }^{1,2}$

\begin{abstract}
${ }^{1}$ Thoracic Oncology Service, Division of Solid Tumor Oncology, Department of Medicine, Memorial Sloan Kettering Cancer Center, New York, New York 10065, USA; ${ }^{2}$ Department of Medicine, Weill Cornell Medical College, New York, New York 10065, USA; ${ }^{3}$ Department of Biostatistics, ${ }^{4}$ Department of Pathology, ${ }^{5}$ Department of Radiology, Memorial Sloan Kettering Cancer Center, New York, New York 10065, USA
\end{abstract}

Corresponding author: rielyg@mskcc.org

(C) 2019 Drilon et al. This article is distributed under the terms of the Creative Commons Attribution-NonCommercial License, which permits reuse and redistribution, except for commercial purposes, provided that the original author and source are credited.

Ontology term: lung adenocarcinoma

Published by Cold Spring Harbor Laboratory Press

doi:10.1101/mcs.a003665
Abstract KRAS G12D-mutant/p53-deficient non-small-cell lung cancer (NSCLC) models are dependent on the NF-kB pathway that can be down-regulated by the proteasome inhibitor bortezomib. Two exceptional responders were observed on prior clinical trials of bortezomib, both of whom had KRAS G12D-mutant NSCLC, prompting the initiation of this single-center phase 2 trial. Patients with advanced KRAS G12D-mutant NSCLC were eligible. Bortezomib was administered at $1.3 \mathrm{mg} / \mathrm{m}^{2}$ subcutaneously (days $1,4,8,11$; 21 -d cycle) until progression or unacceptable toxicity. The primary objective was best objective response (RECIST v1.1). Sixteen patients with KRAS G12D-mutant lung adenocarcinomas were treated. Patients had a median pack year smoking history of 4 (range 0-45). A partial response (PR) was observed in one patient ( $-66 \%$ from baseline) and stable disease in five patients on the first stage of this study (overall response rate of $6 \%, 95 \% \mathrm{Cl}: 0.2-30.2$ ), and further patients were not accrued. The median progression-free survival was $1 \mathrm{mo}(95 \%$ $\mathrm{Cl}$ : 1-6). The median overall survival was $13 \mathrm{mo}$ (95\% Cl: 6-NA). The most common treatment-related adverse events were fatigue (38\%) and diarrhea (26\%). TP53 status did not predict response on exploratory testing. Of note, the patient with a PR had a unique subtype of lung adenocarcinoma_invasive mucinous adenocarcinomas (IMA)—and had rapid clinical improvement and substantial disease regression, which was also previously observed in two other patients with advanced KRAS G12D-mutant lung cancer with IMAs who received bortezomib on separate clinical trials. Exceptional responses to bortezomib can be achieved in KRAS G12D-mutant NSCLCs. KRAS G12D mutation alone, however, is not a robust predictor of response. Further evaluation should only be performed after further elucidation of other factors such as co-occurring alterations and histologic subtype such as IMA that may predict sensitivity to therapy.

[Supplemental material is available for this article.]

${ }^{6}$ These authors contributed equally to this work. 


\section{INTRODUCTION}

Although KRAS mutations are identified in $~ 25 \%$ of lung adenocarcinomas, attempts to develop targeted therapies for this genomic subset of patients have been largely unsuccessful (Kris et al. 2014). It is now well recognized that KRAS-mutant tumors are molecularly heterogeneous (Yu et al. 2015) with biologically distinct subgroups that may require differing approaches to therapy (Skoulidis et al. 2015; Riely et al. 2016). We demonstrated that the KRAS G12D transition mutation is the most common KRAS mutation in patients with minimal to no prior tobacco exposure (Riely et al. 2008) accounting for $58 \%$ of all cases of patients with lung adenocarcinomas in never smokers (Dogan et al. 2012).

Previous preclinical data have established a dependency of KRAS G12D-mutant lung cancer models on the nuclear factor-kappa B (NF-kB) pathway (Fig. 1A). NF-kB is a

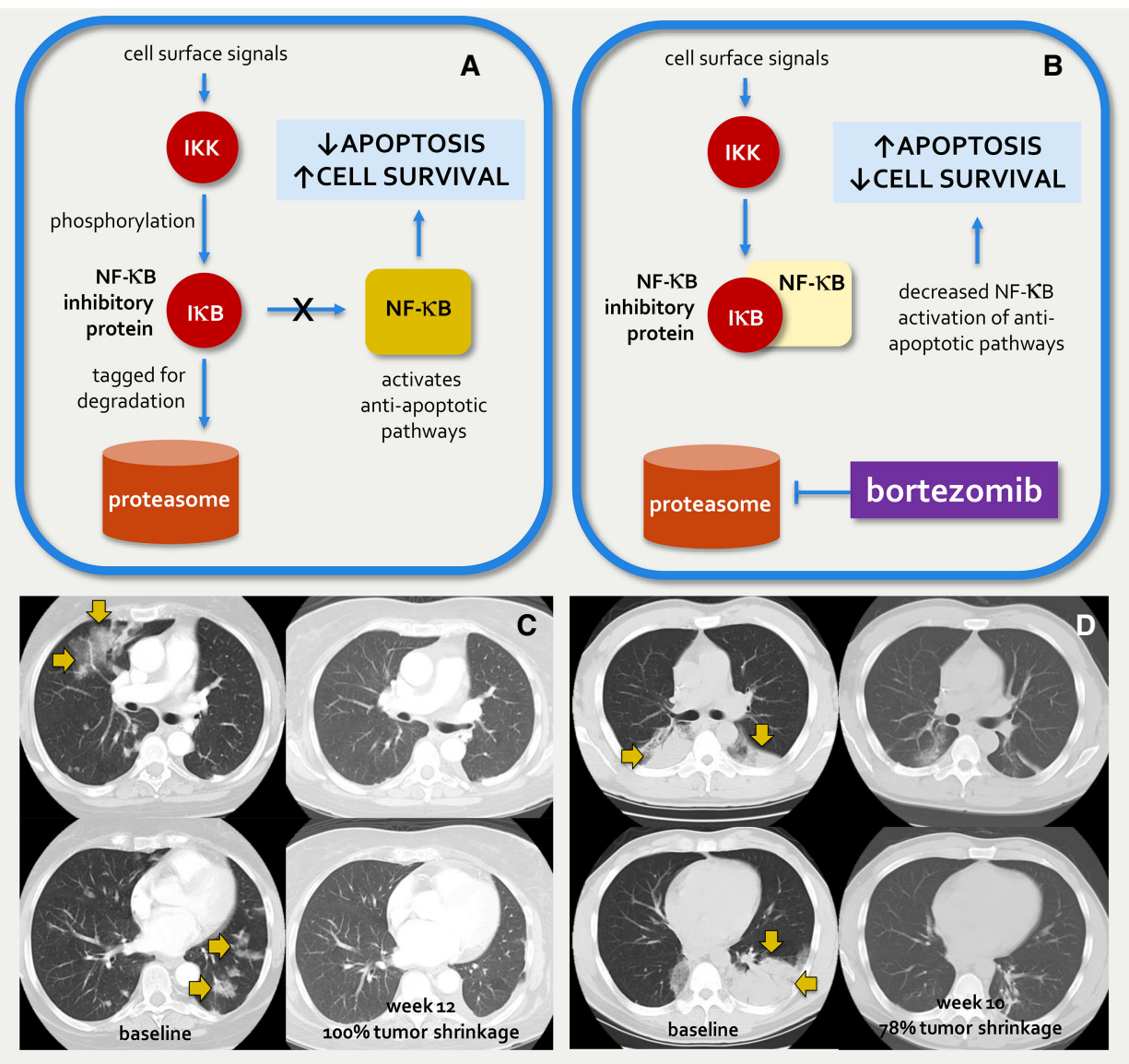

Figure 1. Rationale for bortezomib in KRAS G12D-mutant lung cancers. KRAS G12D-mutant lung cancer models are reliant on NF- $\mathrm{kB}$ pathway activity as shown in $A$. Cell surface signals cause phosphorylation of NF- $\kappa B$ inhibitory proteins by a noncanonical I $\mathrm{KB}$ kinase (IKK or TKB1). Phosphorylation tags these proteins for degradation in the proteasome, thus allowing the uninhibited NF- $\mathrm{kB}$ complex to induce anti-apoptotic pathways. (B) Inhibition of the proteasome by the small molecule bortezomib results in decreased NF-kB inhibitory protein degradation and increased NF-kB inhibition. The consequent decrease in NF-kB-mediated apoptosis leads to increased cell survival. Exceptional responders were observed on separate trials of bortezomib (C, ClinicalTrials.gov number NCT00117351; $D$, inset) for patients with advanced non-small-cell lung cancers. Molecular profiling via Sanger sequencing subsequently revealed that tumor from both patients harbored a KRAS G12D exon 2 mutation. 
transcription complex that induces the expression of genes that play important anti-apoptotic and mitogenic roles. In the context of malignancy, NF-kB can function to protect cancer cells from programmed cell death and drive cellular proliferation (Luo et al. 2009; Sun 2011). More importantly, inhibition of the NF-kB pathway in KRAS G12D-mutant lung cancer models resulted in cancer cell death (Fig. 1B). TKB1 is a noncanonical $I_{\kappa} B$ kinase that positively regulates NF-kB. Barbie et al. (2009) identified TKB1 as a synthetic lethal partner of oncogenic KRAS. They demonstrated that KRAS-mutant lung adenocarcinoma cell lines were highly reliant on NF-KB, and that shRNA knockdown of TKB1 resulted in the induction of apoptosis. Consistent with these results, Xue et al. (2011) showed that the down-regulation of the NF-kB pathway via proteasome inhibition rapidly induced apoptosis in a KRAS-mutant murine lung adenocarcinoma model. The proteasome inhibitor bortezomib induced tumor regression in $K R A S^{\text {LSL-G12D/wt }} ;$ p53 $3^{\text {flox/flox }}$ mice but not in KRAS $S^{\text {LLL-G12D/wt }}$ mice.

We previously observed exceptional responses to bortezomib in patients with advanced non-small-cell lung cancers (NSCLC). A 68-yr-old female never smoker with recurrent lung adenocarcinoma previously treated with gefitinib, erlotinib, and pemetrexed was enrolled into a phase 2 study of intravenous bortezomib (ClinicalTrials.gov number NCT00117351). A confirmed partial response (PR) to therapy was achieved with complete resolution of the patient's target lesions (Fig. 1C). A 42-yr-old male never smoker with recurrent lung adenocarcinoma heavily pretreated with carboplatin and paclitaxel, gemcitabine, mitomycin and vinblastine, docetaxel, and methotrexate was enrolled into a phase 1 trial of intravenous bortezomib (Aghajanian et al. 2002). A rapid clinical response was achieved with resolution of disease-related cough, and serial imaging revealed a PR to therapy and a substantial decrease in bilateral pulmonary masses (Fig. 1D). Both patients discontinued therapy after $\sim 3$ mo because of bortezomib-related peripheral neuropathy. Molecular profiling via Sanger sequencing revealed a KRAS G12D mutation in tumors from both patients. In addition, the histologic subtype of both adenocarcinomas was invasive mucinous adenocarcinomas (IMA) (former "mucinous bronchioloalveolar carcinoma").

To further evaluate these findings, we initiated a prospective phase 2 trial of bortezomib for patients with KRAS G12D-mutant lung cancers with the intent of determining the activity of the drug in this molecularly enriched population. Because of our prior experience with drug-related neuropathy limiting bortezomib administration in both exceptional responders, subcutaneous bortezomib was chosen over intravenous bortezomib given the reported lower incidence of neuropathy in the former (Moreau et al. 2011).

\section{RESULTS}

\section{Subcutaneous Bortezomib Activity and Safety}

Sixteen patients with stage IV KRAS G12D-mutant lung cancers were accrued to this trial and treated with bortezomib (Table 1). Patients were either never $(38 \%, n=6 / 16)$ or former $(62 \%$, $n=10 / 16$ ) cigarette smokers with a median pack year history of 4 (range $0-45$ ) pack years. There was a slight male predominance $(56 \%, n=9 / 16)$, and the majority of patients were Caucasian $(81 \%, n=13 / 16)$. All patients had lung adenocarcinoma, of which $44 \%(7 / 16)$ were IMA. The median number of prior systemic therapies for advanced disease was 2 (range 1-4).

Of the 16 patients accrued to the first stage of this study, only one confirmed PR was observed. Complete responses were not observed. SD was achieved in five patients, and a best response of disease progression was noted in 10 patients. Of the 16 patients, only 15 were evaluable for radiologic response to bortezomib as one patient discontinued therapy prior to his first on-treatment imaging assessment because of disease progression. The waterfall plot of best objective response to bortezomib in 15 patients with KRAS G12D-mutant lung cancers and response-evaluable disease is shown in Figure 2. 


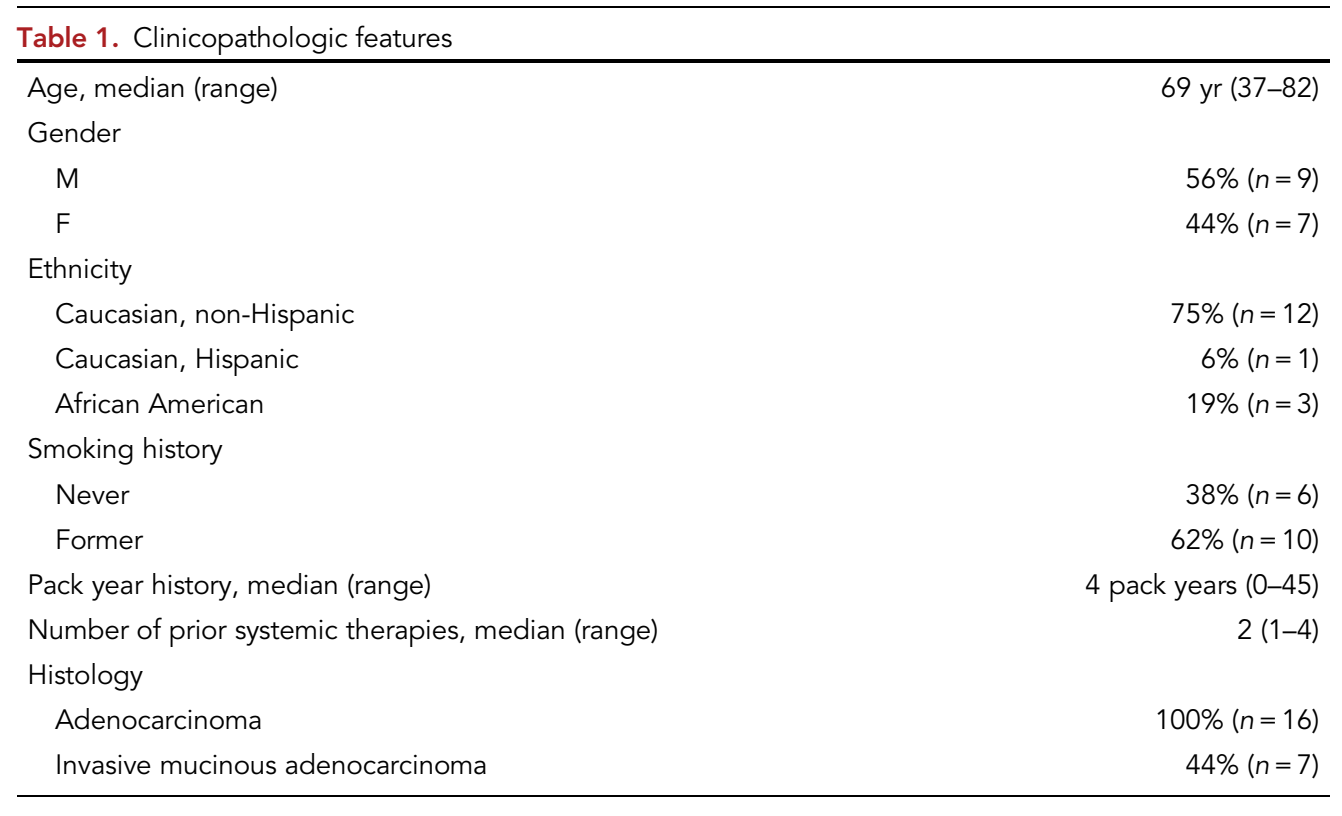

The demographics, stage, smoking history, number of prior systemic therapies, and histology of 16 patients with KRAS G12D-mutant lung adenocarcinomas treated with bortezomib on this phase 2 trial are summarized.

The overall response rate was $6 \%(95 \% \mathrm{Cl}: 0.2-30.2, n=1 / 16)$. The disease control rate was 31\% (95\% Cl: 11.0-58.7, $n=5 / 16)$. The duration of bortezomib therapy is shown in Supplemental Figure S1. Long-term disease control (lasting $>6 \mathrm{mo}$ ) was achieved in two patients. The first patient was a 37-yr-old female never smoker who received cisplatin, pemetrexed, and bevacizumab, cisplatin and paclitaxel, and durvalumab with clear PD on the latter prior to enrolling into this phase 2 trial. SD was achieved (best objective response of $13 \%$ disease shrinkage) and maintained for $17 \mathrm{mo}$, after which bortezomib was discontinued because of disease progression. The second patient was a 79-yr-old female never smoker. SD was achieved and maintained for 8 mo.

The median PFS was $1.4 \mathrm{mo}$ (95\% Cl: 1.2-8.4 mo) and the median OS was $13.4 \mathrm{mo}$ (95\% $\mathrm{Cl}$ : 6.4-NA mo). Kaplan-Meier curves for PFS and OS are shown in Supplemental Figure S2. The most common drug-related adverse events were fatigue (38\%), diarrhea (26\%), anorexia $(25 \%)$, constipation (25\%), nausea (25\%), and papulopustular rash (25\%). Injection site reactions were observed in two patients (13\%). Peripheral sensory neuropathy was likewise observed in two patients (13\%). Only grade 1 and 2 (and no grade 3, 4, or 5) drug-related adverse events were observed as outlined in Table 2. In nine patient cases, there was sufficient tissue to perform next-generation sequencing and the identified mutations are provided in the variant tables (Table 3, Supplemental Table S1).

\section{Characteristics of Patient with PR}

Although bortezomib did not induce responses in the majority of patients with KRAS G12Dmutant lung adenocarcinomas on this phase 2 trial, dramatic disease shrinkage was observed in an exceptional responder. An 80-yr-old woman never smoker presented with a cough and a left lower lobe lung mass in 2011. A biopsy revealed lung IMA, and she subsequently underwent a left lower lobectomy and mediastinal dissection remarkable for pT2NOMO (stage IB) disease. Adjuvant therapy was not administered. Five months after her surgery, she was found to have recurrent disease involving the lung, and she was treated with carboplatin and pemetrexed followed by maintenance pemetrexed, and later 

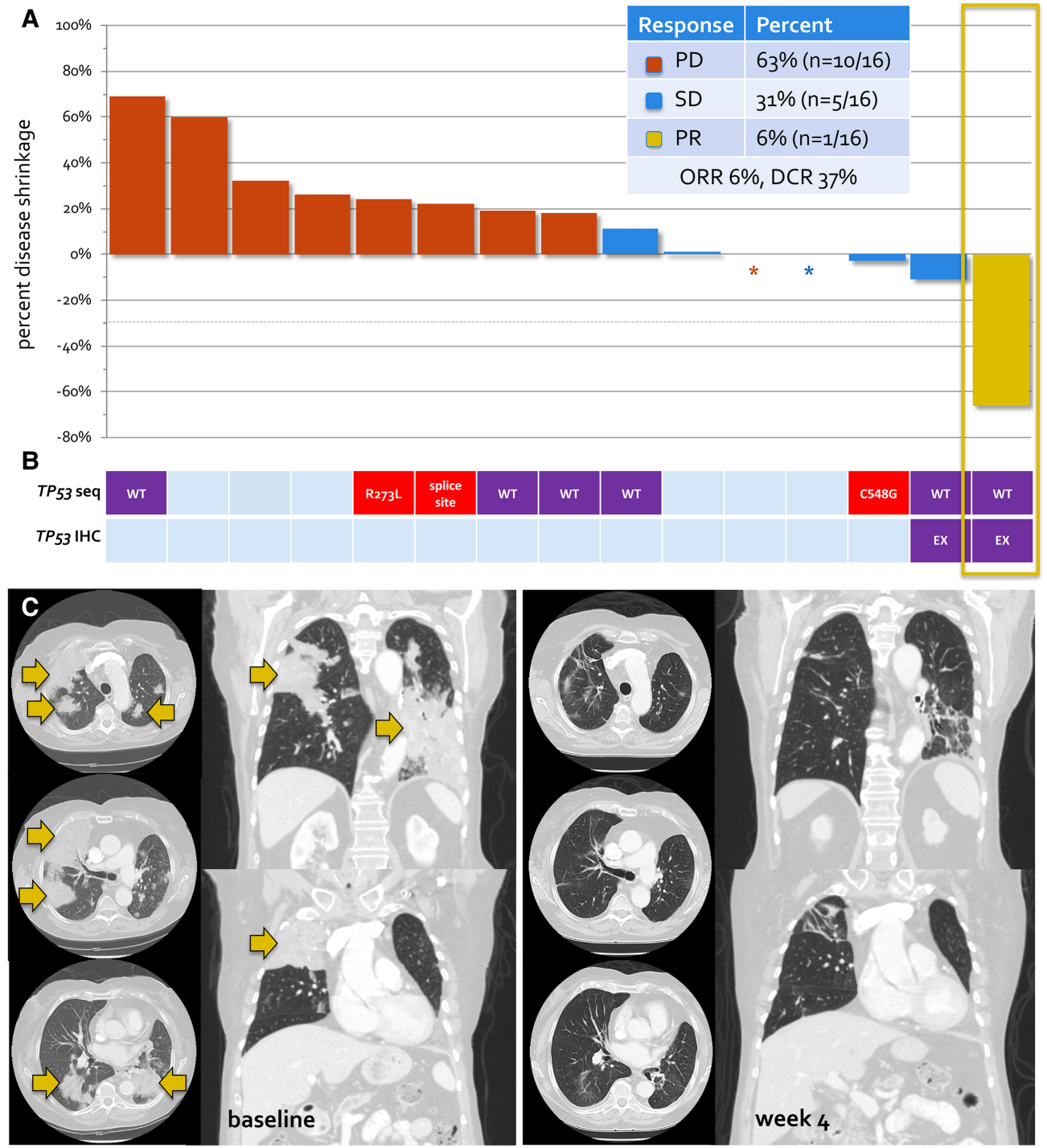

Figure 2. Activity of bortezomib in KRAS G12D-mutant lung cancers. (A) The waterfall plot of best objective response to bortezomib. Of 15 evaluable patients treated with bortezomib, one patient had a dramatic response to therapy (yellow box), consistent with previously observed cases. (B) An exploratory analysis of TP53 status was performed by tumor DNA sequencing (TP53 seq) and immunohistochemistry (TP53 IHC) in cases with available tissue. Patients with wild-type TP53 gene or nonaberrant protein expression (purple) or TP53-expressing tumors are shown in addition to patients with TP53-mutant (red) disease. Patients with insufficient tissue are shown in light blue. TP53 status did not predict response to bortezomib. (C) Serial imaging of an exceptional responder to bortezomib. A brisk and dramatic response to therapy with $66 \%$ disease shrinkage and substantial clearing of bilateral areas of tumor consolidation was achieved. (PD) Progression of disease; (SD) stable disease; (PR) partial response; (ORR) overall response rate; (DCR) disease control rate.

gemcitabine after progression of disease. Molecular profiling via Sanger sequencing revealed a KRAS G12D mutation and the patient was enrolled into this study.

A PR (66\% reduction in disease burden) was observed after 6 wk of therapy with bortezomib and confirmed at $12 \mathrm{wk}$. Rapid reduction in the size of bilateral pulmonary masses (Fig. 2) was accompanied by a clinical response to therapy with decreasing disease-related 


\begin{tabular}{lcccc}
\hline Table 2. Treatment-related adverse events & \multicolumn{3}{c}{ Grade, $n$ (\%) } \\
\cline { 2 - 5 } & Grade 1 & Grade 2 & Grade 3/4 & All grades \\
\hline Fatigue & $2(13 \%)$ & $4(25 \%)$ & - & $6(38 \%)$ \\
Anorexia & $4(25 \%)$ & $0(0 \%)$ & - & $4(25 \%)$ \\
Constipation & $3(19 \%)$ & $1(6 \%)$ & - & $4(25 \%)$ \\
Diarrhea & $2(13 \%)$ & $2(13 \%)$ & - & $4(26 \%)$ \\
Nausea & $4(25 \%)$ & - & - & $4(25 \%)$ \\
Papulopustular rash & $4(25 \%)$ & - & - & $4(25 \%)$ \\
Headache & $3(19 \%)$ & - & - & $3(19 \%)$ \\
Abdominal distension & $2(13 \%)$ & - & - & $2(13 \%)$ \\
Injection site reaction & $2(13 \%)$ & - & - & $2(13 \%)$ \\
Mucositis oral & $2(13 \%)$ & - & - & $2(13 \%)$ \\
Peripheral sensory neuropathy & $1(6 \%)$ & $1(6 \%)$ & - & $2(13 \%)$ \\
Thrombocytopenia & $1(6 \%)$ & $1(6 \%)$ & - & $2(13 \%)$ \\
Dry mouth & $1(6 \%)$ & - & - & $1(6 \%)$ \\
Fever & $1(6 \%)$ & - & - & $1(6 \%)$ \\
Flu-like symptoms & $1(6 \%)$ & - & - & $1(6 \%)$ \\
Localized edema & $1(6 \%)$ & - & - & $1(6 \%)$ \\
Pain in extremity & $0(0 \%)$ & $1(6 \%)$ & - & $1(6 \%)$ \\
Vomiting & $1(6 \%)$ & & - & $1(6 \%)$ \\
\hline
\end{tabular}

dyspnea. The patient developed intolerable drug-related grade 2 peripheral neuropathy and therapy was discontinued after 5 mo.

\section{p53 Status and Response to Therapy}

Following the preclinical observation that significant tumor regression and prolonged survival were only observed in KRAS G12D models with p53 deficiency (KRAS ${ }^{\text {LSL-G12D/wt; }}$ p53 $3^{\text {flox/flox }}$ mice) compared to p53-intact models (KRAS $S^{\text {LSL-G12D/wt }}$ mice) (Xue et al. 2011), we performed an exploratory analysis of p53 status sequencing and immunohistochemistry.

Contrary to what might have been predicted by preclinical data, TP53 was not found to be mutated or p53 aberrantly expressed in an examination of tumor from the exceptional responder on this trial. Next-generation sequencing confirmed the presence of a KRAS G12D mutation in addition to CDKN2Ap16INK4A R80* and CDKN2Ap14ARF mutations, but no mutations in TP53 were identified (Table 3). Additionally, immunohistochemical analysis revealed focal labeling for p53 in $20 \%$ of tumor cells, which is consistent with a wild-type TP53 pattern. Similarly, the longest responder who remained on bortezomib for 16.8 mo with SD had TP53 wild-type disease without aberrant p53 expression by immunohistochemistry. This patient also was found to have an MLL2 mutation (Table 3). TP53 status for other

\begin{tabular}{lcccccc}
\hline Table 3. Variant table & HGVS & HGVS & DNA & Protein \\
Gene & Chromosome & $\begin{array}{c}\text { Variant } \\
\text { reference }\end{array}$ & $\begin{array}{c}\text { Predicted effect } \\
\text { (substitution, } \\
\text { reference }\end{array}$ & $\begin{array}{c}\text { Genotype } \\
\text { type }\end{array}$ & $\begin{array}{c}\text { (heterozygous/ } \\
\text { homozygous) }\end{array}$ \\
\hline KRAS & 12 & c.35G $>$ A & p.G12D & Missense & Substitution & Heterozygous \\
\hline
\end{tabular}


patients with available tissue for sequencing and immunohistochemistry is summarized in Figure 2 and Table 3. No responses were observed in three patients with KRAS G12Dmutant and TP53-mutant disease.

\section{DISCUSSION}

Although bortezomib was inactive in the majority of patients with KRAS G12D-mutant lung cancers on this negative phase 2 trial, exceptional response to therapy was observed. Rapid clinical improvement and substantial disease regression were achieved in one patient with a confirmed response on this trial, similar to that previously observed in two other patients with advanced KRAS G12D-mutant lung adenocarcinomas who received bortezomib on separate clinical trials. All three patients were never smokers with advanced lung adenocarcinomas. Remarkably, all three patients had a unique subtype of lung adenocarcinoma-invasive mucinous adenocarcinomas (IMA). Such tumors are characterized by distinct histology (mucinous cells with prominent lepidic growth pattern) and highly distinct genomic profile, dominated by KRAS G12D mutations as well as recently described NRG1 and other fusions that are specific to this tumor type (Shim et al. 2015). These tumors represent $~ 7 \%$ of unselected lung adenocarcinomas. The marked overrepresentation in this series is likely due to preselection for KRAS G12D mutations.

These results support the observation that KRAS-mutant lung cancers represent a highly heterogeneous group of tumors. Beyond differences in mutation profile, with KRAS G12C transversion mutations occurring more commonly in smokers and KRAS G12D mutations occurring more often in never smokers, the outlier responses observed with bortezomib in KRAS G12D-mutant lung cancers point to an additional level of heterogeneity within molecularly enriched and mutation-specific groups of tumors. The biology responsible for the outlier responses observed with bortezomib remains unclear. Prior in vivo data on response to bortezomib showed that tumor regressions and prolonged survival were largely observed in mice whose tumors were both KRAS G12D-mutant and p53-deficient, in contrast to those whose tumors were KRAS G12D-mutant and TP53 wild-type (Xue et al. 2011). An exploratory analysis of p53 status on this trial, however, did not reveal TP53 mutation or the lack of p53 expression in tumor from the exceptional responder or from the patient with long-term disease control, suggesting other unmeasured factors accounting for differential response.

Post hoc next-generation sequencing also did not reveal a clear mechanism of response or resistance. This may in part be due to the limited sequencing results available and number of patients with sufficient tissue for testing. However, the patients profiled had a variety of different co-occurring alterations, further emphasizing the heterogeneity of KRAS-mutant lung cancer. Recent studies have found that concurrent alterations such as KEAP1 and STK11 in KRAS-mutant lung cancer are associated with distinct clinical behavior and worse clinical outcomes (Aghajanian et al. 2002; Dogan et al. 2012; Skoulidis et al. 2015; Schabath et al. 2016; Facchinetti et al. 2017). Future trials may require stratification by these co-occurring mutations to reliably assess response to therapy.

Future investigations of NF-kB pathway inhibitors in KRAS G12D-mutant lung adenocarcinomas should also only be performed with the requirement that tumors be comprehensively sequenced. Comprehensive molecular profiling via next-generation sequencing with MSK-IMPACT was not available when this trial was launched at our institution, and the lack of tissue to determine p53 status in a large proportion of patients underscores the challenges encountered with post hoc exploratory testing. Despite the use of subcutaneous bortezomib on this trial, drug-related peripheral sensory neuropathy remained prohibitive of continued dosing, and alternative dosing schedules and other NF-kB pathway modifiers should be explored in the clinic. 
We strongly believe that further investigation into the biology behind response to NF-kB pathway inhibition in KRAS G12D-mutant models should be conducted given the outlier responses observed, with close attention paid to concurrent genomic events beyond TP53 alterations that may serve as modifiers of targeted therapy activity. To date, no targeted therapy has been approved for KRAS-mutant lung cancers, and should a predictive combination of biomarkers beyond KRAS G12D mutation be discovered, novel proteasome or NF-kB inhibitors may offer an additional systemic therapeutic beyond chemotherapy and immunotherapy for this subset of patients.

\section{METHODS}

\section{Study Population}

Patients were eligible for participation if they met the following criteria: pathologic evidence of NSCLC, advanced (stage IIIB/IV) disease, tumor with a KRAS mutation, Karnofsky performance status $\geq 70 \%$, age $\geq 18 \mathrm{yr}$, received one prior line of therapy, and measurable disease by Response Evaluation Criteria in Solid Tumors (RECIST) version 1.1. In addition, patients had to either be never smokers (history of smoking $<100$ lifetime cigarettes) or have tumor harboring a KRAS G12D mutation regardless of smoking history. KRAS mutation testing was performed via multiplex hotspot polymerase chain reaction mass spectrometry (Sequenom).

Patients with uncontrolled metastatic disease involving the central nervous system, at least grade 2 peripheral neuropathy (CTCAE version 4.0), and hypersensitivity to boron or mannitol were excluded. The presence of an unstable medical condition and the presence of an active concurrent malignancy with the exception of in situ cancers, completely resected basal cell or squamous cell carcinoma of the skin, and low-risk prostate cancer after initial curative therapy were exclusionary.

\section{Design}

This was a single-institution, open-label, phase 2 clinical trial (NCT 01833143). The primary objective of this study was to determine the response rate of subcutaneous bortezomib in patients with advanced, KRAS-mutant NSCLCs. Secondary objectives included an evaluation of progression-free survival (PFS), overall survival (OS), and toxicity. Bortezomib was administered subcutaneously in 21-d cycles at a dose of $1.3 \mathrm{mg} / \mathrm{m}^{2}$ on days $1,4,8$, and 11 . Patients received antiviral prophylaxis for herpes zoster with acyclovir at $400 \mathrm{mg}$ daily. Treatment was continued until disease progression, treatment delay of more than $2 \mathrm{wk}$, or unacceptable toxicity. Dose interruption and modification was permitted via a standard algorithm. Only two dose reductions to $1.0 \mathrm{mg} / \mathrm{m}^{2}$ and $0.7 \mathrm{mg} / \mathrm{m}^{2}$, respectively, were allowed. Dose re-escalation was not permitted. Computed tomography (CT) of the chest, abdomen, and pelvis was performed at baseline and every $6 \mathrm{wk}$. Objective response was classified as a $\mathrm{PR}$, stable disease (SD), or progressive disease (PD).

\section{Statistics}

The primary endpoint of this study was best objective response to bortezomib, evaluated at any time between treatment initiation and documented progression of disease. A Simon two-stage minimax design was used to test the null hypothesis of a $10 \%$ objective response rate (ORR) against the desired alternative of a 30\% ORR with a one-sided type I error of 10\% and a power of $90 \%$. The null hypothesis was based on historical data of a best objective response of $10 \%$ of NSCLCs to cytotoxic therapy after failure of first-line chemotherapy.

In the first stage of the design, 16 patients are accrued. If less than two responses are observed, the study is terminated and declared negative. If at least two responses are observed, 
Competing Interest Statement

G.J.R.'s institution receives clinical research support from Pfizer, Takeda, Novartis, Merck, and Infinity. He has been a compensated consultant for Genentech/Roche. V.M. is an employee of Foundation Medicine, Inc. A.D. is a consultant for Ignyta, LOXO Oncology, TP Therapeutics, AstraZeneca, Pfizer, Blueprint Medicines, Genentech/Roche, and Takeda and has received research funding from Foundation Medicine. M.L. has received advisory board compensation from Boehringer Ingelheim, AstraZeneca, BristolMyers Squibb, Takeda, and Bayer and research support from LOXO Oncology and Helsinn Healthcare. M.A. is a consultant for AstraZeneca and received support from AstraZeneca, Invivoscribe, and RainDance Technologies. H.A.Y. has consulted for AstraZeneca and has research funding to her institution from AstraZeneca, Lilly, Pfizer, Novartis, and Daiichi. K.C.A. has been a compensated consultant for AstraZeneca.

\section{Referees}

Ferdinandos Skoulidis

Anonymous

Received November 12, 2018; accepted in revised form January 16, 2019. an additional nine patients are accrued to the second stage. At the end of the study, if five or more patients respond out of a total of 25 patients enrolled, the treatment is considered worthy of further investigation. Patients who received at least one dose of bortezomib were evaluable for response. For patients who discontinued treatment before documented radiologic progression, every effort was made to perform imaging prior to study discontinuation. If this was not possible, patients were considered nonresponders.

PFS and OS were calculated using Kaplan-Meier estimators starting from the time of treatment initiation. Patients were followed until progression of disease or death for PFS, or until death for OS. Patients who did not experience the event of interest were censored at the time of the last available follow-up. Eight-week SD was summarized using descriptive statistics.

\section{Molecular Profiling}

Initial profiling for KRAS was performed via Sanger sequencing. An exploratory analysis of TP53 status and other concurrent mutations was performed by next-generation sequencing using lon Torrent in cases with sufficient tissue. Testing was performed using a custom AmpliSeq panel to amplify all coding regions of 98 cancer associated genes followed by next-generation sequencing. Briefly, genomic DNA was extracted from tumor and sequence libraries were prepared through a series of enzymatic steps including multiplex amplification, partial digestion, ligation of barcoded sequence adaptors, and clonal PCR amplification by emulsion. Purified DNA fragments were sequenced on an lon Torrent Personal Genome Machine, PGM, as paired-end reads and subjected to a custom analysis pipeline to identify somatic mutations.

Immunohistochemistry for p53 was performed on samples with available tissue using a D07 antibody (mouse monoclonal, Ventana). The presence of aberrant p53 expression, indicative of mutant TP53, was assessed according to the established criteria, which included either complete loss of expression or diffuse overexpression (Yemelyanova et al. 2011).

\section{CONCLUSION}

The proteasome inhibitor bortezomib is inactive in the majority of patients with advanced KRAS G12D-mutant lung adenocarcinomas; however, dramatic and durable clinical benefits have been observed in a small number of select patients. The molecular basis behind the exceptional responses observed with bortezomib should continue to be explored, as should novel inhibitors of the NF-kB pathway on clinical trials where prospective comprehensive molecular profiling is performed.

\section{ADDITIONAL INFORMATION}

\section{Data Deposition and Access}

The KRAS G12D variant was submitted to ClinVar (http://www.ncbi.nlm.nih.gov/clinvar/) and can be found under accession number SCV000882686.

\section{Ethics Statement}

This study was approved by the IRB at Memorial Sloan Kettering Cancer Center. Appropriate patient consent was obtained prior to participation in this study. 


\section{Author Contributions}

A.D. and A.J.S. share first authorship. A.D., A.J.S., V.M., and G.J.R. were responsible for conception and design of this study. A.D., A.J.S., K.C.A., A.L., J.M., H.A.Y., E.P., M.K., A.H.-S., V.M., M.G., M.L., M.A., N.R., M.G.K., G.J.R. were involved in acquisition and management of patients and data. A.D., A.J.S., G.J.R., A.N., M.A., M.L. were involved in analysis and interpretation of data. All authors were involved in writing, reviewing, and revising the manuscript. The study was supervised by G.J.R. and A.D.

\section{Funding}

This research was supported in part by Millennium, an ASCO Young Investigator Award (to A.D.), and the National Cancer Institute (NCI) (P30 P30CA008748).

\section{REFERENCES}

Aghajanian C, Soignet S, Dizon DS, Pien CS, Adams J, Elliott PJ, Sabbatini P, Miller V, Hensley ML, Pezzulli S, et al. 2002. A phase I trial of the novel proteasome inhibitor PS341 in advanced solid tumor malignancies. Clin Cancer Res 8: 2505-2511.

Barbie DA, Tamayo P, Boehm JS, Kim SY, Moody SE, Dunn IF, Schinzel AC, Sandy P, Meylan E, Scholl C, et al 2009. Systematic RNA interference reveals that oncogenic KRAS-driven cancers require TBK1. Nature 462: 108-112. doi:10.1038/nature08460

Dogan S, Shen R, Ang DC, Johnson ML, D’Angelo SP, Paik PK, Brzostowski EB, Riely GJ, Kris MG, Zakowski MF, et al. 2012. Molecular epidemiology of EGFR and KRAS mutations in 3,026 lung adenocarcinomas: higher susceptibility of women to smoking-related KRAS-mutant cancers. Clin Cancer Res 18: 61696177. doi:10.1158/1078-0432.CCR-11-3265

Facchinetti F, Bluthgen MV, Tergemina-Clain G, Faivre L, Pignon JP, Planchard D, Remon J, Soria JC, Lacroix L, Besse B. 2017. LKB1/STK11 mutations in non-small cell lung cancer patients: descriptive analysis and prognostic value. Lung Cancer 112: 62-68. doi:10.1016/j.lungcan.2017.08.002

Kris MG, Johnson BE, Berry LD, Kwiatkowski DJ, lafrate AJ, Wistuba II, Varella-Garcia M, Franklin WA, Aronson $\mathrm{SL}$, Su PF, et al. 2014. Using multiplexed assays of oncogenic drivers in lung cancers to select targeted drugs. JAMA 311: 1998-2006. doi:10.1001/jama.2014.3741

Luo J, Emanuele MJ, Li D, Creighton CJ, Schlabach MR, Westbrook TF, Wong KK, Elledge SJ. 2009. A genome-wide RNAi screen identifies multiple synthetic lethal interactions with the Ras oncogene. Cell 137: 835-848. doi:10.1016/j.cell.2009.05.006

Moreau P, Pylypenko H, Grosicki S, Karamanesht I, Leleu X, Grishunina M, Rekhtman G, Masliak Z, Robak T, Shubina A, et al. 2011. Subcutaneous versus intravenous administration of bortezomib in patients with relapsed multiple myeloma: a randomised, phase 3, non-inferiority study. Lancet Oncol 12: 431-440. doi:10 .1016/S1470-2045(11)70081-X

Riely GJ, Kris MG, Rosenbaum D, Marks J, Li A, Chitale DA, Nafa K, Riedel ER, Hsu M, Pao W, et al. 2008. Frequency and distinctive spectrum of KRAS mutations in never smokers with lung adenocarcinoma. Clin Cancer Res 14: 5731-5734. doi:10.1158/1078-0432.CCR-08-0646

Riely GJ, Jordan E, Kim HR, Yu HA, Berger MF, Solit DB, Kris MG, Ni A, Arcila ME, Ladanyi M. 2016. Association of outcomes and co-occurring genomic alterations in patients with KRAS-mutant non-small cell lung cancer. J Clin Oncol 34: 9019-9019. doi:10.1200/JCO.2016.34.15_suppl.9019

Schabath MB, Welsh EA, Fulp WJ, Chen L, Teer JK, Thompson ZJ, Engel BE, Xie M, Berglund AE, Creelan BC, et al. 2016. Differential association of STK11 and TP53 with KRAS mutation-associated gene expression, proliferation and immune surveillance in lung adenocarcinoma. Oncogene 35: 3209-3216. doi:10.1038/ onc. 2015.375

Shim HS, Kenudson M, Zheng Z, Liebers M, Cha YJ, Hoang Ho Q, Onozato M, Phi Le L, Heist RS, lafrate AJ. 2015. Unique genetic and survival characteristics of invasive mucinous adenocarcinoma of the lung. $J$ Thorac Oncol 10: 1156-1162. doi:10.1097/JTO.0000000000000579

Skoulidis F, Byers LA, Diao L, Papadimitrakopoulou VA, Tong P, Izzo J, Behrens C, Kadara H, Parra ER, Canales $J R$, et al. 2015. Co-occurring genomic alterations define major subsets of KRAS-mutant lung adenocarcinoma with distinct biology, immune profiles, and therapeutic vulnerabilities. Cancer Discov 5: 860-877. doi:10.1158/2159-8290.CD-14-1236

Sun SC. 2011. Non-canonical NF-kB signaling pathway. Cell Res 21: 71-85. doi:10.1038/cr.2010.177 
Xue W, Meylan E, Oliver TG, Feldser DM, Winslow MM, Bronson R, Jacks T. 2011. Response and resistance to NF-kB inhibitors in mouse models of lung adenocarcinoma. Cancer Discov 1: 236-247. doi:10.1158/21598290.CD-11-0073

Yemelyanova A, Vang R, Kshirsagar M, Lu D, Marks MA, Shih le M, Kurman RJ. 2011. Immunohistochemical staining patterns of p53 can serve as a surrogate marker for TP53 mutations in ovarian carcinoma: an immunohistochemical and nucleotide sequencing analysis. Mod Pathol 24: 1248-1253. doi:10.1038/modpa thol.2011.85

Yu HA, Sima CS, Shen R, Kass S, Gainor J, Shaw A, Hames M, lams W, Aston J, Lovly CM, et al. 2015. Prognostic impact of KRAS mutation subtypes in 677 patients with metastatic lung adenocarcinomas. $J$ Thorac Oncol 10: 431-437. doi:10.1097/JTO.0000000000000432 


\section{COLD SPRING HARBOR Molecular Case Studies}

\section{Exceptional responders with invasive mucinous adenocarcinomas: a phase 2 trial of bortezomib in patients with KRAS G12D-mutant lung cancers}

Alexander Drilon, Adam J. Schoenfeld, Kathryn C. Arbour, et al.

Cold Spring Harb Mol Case Stud 2019, 5: a003665

Access the most recent version at doi: $10.1101 / \mathrm{mcs} . a 003665$

Supplementary http://molecularcasestudies.cshlp.org/content/suppl/2019/03/25/mcs.a003665.D
Material

References This article cites 16 articles, 5 of which can be accessed free at: http://molecularcasestudies.cshlp.org/content/5/2/a003665.full.html\#ref-list-1

License This article is distributed under the terms of the Creative Commons Attribution-NonCommercial License, which permits reuse and redistribution, except for commercial purposes, provided that the original author and source are credited.

Email Alerting Receive free email alerts when new articles cite this article - sign up in the box at the Service top right corner of the article or click here. 